\title{
Criminologie
}

\section{Une question d'actualité : l'intégration scolaire dans les centres d'accueil : impact sur les objectifs poursuivis et le climat social}

\section{Richard E. Tremblay}

Volume 11, numéro 1, 1978

Centres d'accueil du Québec : accréditation et évaluation

URI : https://id.erudit.org/iderudit/017083ar

DOI : https://doi.org/10.7202/017083ar

Aller au sommaire du numéro

Éditeur(s)

Les Presses de l'Université de Montréal

ISSN

0316-0041 (imprimé)

1492-1367 (numérique)

Découvrir la revue

Citer cet article

Tremblay, R. E. (1978). Une question d'actualité : l'intégration scolaire dans les centres d'accueil : impact sur les objectifs poursuivis et le climat social.

Criminologie, 11(1), 65-74. https://doi.org/10.7202/017083ar 


\section{UNE QUESTION D'ACTUALITE : \\ L'INTEGRATION SCOLAIRE \\ DANS LES CENTRES D'ACCUEIL :}

IMPACT SUR LES OBJECTIFS POURSUIVIS

ET LE CLIMAT SOCIAL

Richard E. Tremblay

Depuis la publication du « Guide 》 de la mission du ministère de l'Éducation - ministère des Affaires sociales (MEQ -MAS) pour assurer la scolarisation optimale des enfants des établissements du ministère des Affaires sociales (MEQ - MAS, 1974), le problème de \& l'intégration scolaire » dans les centres d'accueil du Québec est devenu un sujet d'actualité controversé.

Etant donné l'impact probable de l'implantation des directives du \& Guide > sur le traitement des jeunes admis dans les centres d'accueil, il apparaît important de tenter de prévoir les modalités de cet impact de façon à en maximiser les effets positifs et minimiser les effets négatifs.

Dans la présente discussion, deux champs d'impact probable sont étudiés : les objectifs poursuivis par le milieu (le centre d'accueil) et le climat social du milieu. La discussion est basée sur des recherches récentes concernant le traitement d'adolescents délinquants ainsi que sur une réflexion ayant trait à l'histoire des modèles de traitement d'adolescents délinquants.

Bien que la majorité du matériel utilisé pour cette étude provienne d'expériences de traitement d'adolescents délinquants, la discussion apparaît utile pour l'ensemble des établissements (du MAS) pour jeunes.

\section{Les recommandations du \& guide, et l'histoire des modeles de traitement}

Une étude de l'histoire des modèles de traitement de jeunes délinquants permet de constater un certain nombre de « modes » reliés plus ou moins aux grands courants de pensée de chacune des époques.

D'une façon générale, avant le dix-neuvième siècle, les jeunes délinquants étaient incarcérés avec les adultes criminels (Sanders, 1970). L'objectif de l'incarcération étant de mettre à l'écart 
et de punir (le modele «carcéral $»)$. $\lambda$ la fin du dix-huitième et au début du dix-neuvième siècle, particulièrement dans les pays anglophones, des personnes influencées par l'éthique puritaine proposèrent d'aider les jeunes délinquants en les instruisant, en leur faisant apprendre un métier et en leur inculquant une certaine discipline. Le rationnel de cette approche (le modèle « réforme ») étant que la discipline, l'instruction et le métier, permettraient au jeune de se trouver un travail convenable et de résister aux tentations d'actes délinquants (Lehman, 1972, p. 205 ; Sanders, 1970, p. 70-77, 80-86). Le modèle «thérapeutique » apparut au début du vingtième siècle. Les psychiatres organicistes et les psychanalystes (comme plus tard les behavioristes) affirmaient, chacun à sa façon, que le délinquant est un malade et qu'il doit être traité (Lehman, 1972, p. 205 et 207).

Il est assez rare aujourd'hui que des «spécialistes \$ proposent un modèle « carcéral ». Le modèle " réforme» est de moins en moins présenté comme étant «la solution » au problème, mais il est sûrement le modèle le plus utilisé (Dunlop, 1974 ; Street, Vinter et Perrow, 1966). Le modèle « thérapeutique » bien qu'il soit proposé d'une façon claire depuis au moins un demi-siècle, demeure en pratique une exception considérée comme avant-gardiste (Millham, Bullock et Cherrett, 1973, p. 10 ; Hood et Sparks, 1970, p. 215).

Chacun des modèles de traitement a ses caractéristiques propres. Cependant l'étude chronologique des modèles de traitement semble démontrer que le modèle proposé pour remplacer un modèle précédent conserve des caractéristiques du modèle précédent. Par exemple le modèle « réforme » tout en proposant d'instruire, d'initier au travail et de discipliner continuait de proposer le retrait de l'individu de la société (incarcération, ou plutôt institutionalisation). De la même façon le modèle * thérapeutique » tout en proposant de «guérir » (intervenir sur les causes du problème) continue de proposer le retrait de la société, l'utilisation d'activités d'instruction, d'initiation au travail et de discipline.

Récemment un nouveau modèle, ou une modification du modèle thérapeutique (l'histoire est trop récente pour décider), semble influencer les théoriciens et les planificateurs. Le concept de «normalisation ( Wolfensberger, 1972) propose le comportement « normal comme objectif de traitement des personnes 
inadaptées et des moyens « normaux 》 pour atteindre ces objectifs. Pour celui qui connaît mal les services pour personnes inadaptées, le concept de «normalisation » peut apparaître simpliste, voire même naï. Cependant, aux initiés il apparaît comme une réaction salutaire aux exagérations du modèle thérapeutique (Batshaw, et al., 1975, p. 8-10). Il n'en demeure pas moins, cependant, que le concept (le modèle ?) de «normalisation » conserve les caractéristiques des modèles précédents : le retrait de la société est parfois inévitable ; l'instruction, la préparation au travail et la discipline sont des éléments de la vie «normale >; l'intervention sur les causes du problème (de la maladie?) demeure souvent nécessaire.

Le \& Guide \& de la mission MEQ - Mas (1974) ne situe pas ses recommandations par rapport à un ou à des modèles de traitement. Les deux premiers principes généraux, l'accessibilité aux services d'enseignement et l'accessibilité aux services de santé et aux services sociaux, pourraient fort bien avoir été inspirés du principe de «normalisation ». Mais ils pourraient tout aussi bien avoir été inspirés du modèle \& réforme que du modèle « thérapeutique ».

Quelle que soit l'inspiration du «Guide », ce sont les effets de ses recommandations qui nous permettront de juger de leur valeur. Comme les recommandations ont pour premier objectif la scolarisation optimale des jeunes dans les établissements du MAS, le principal danger semble être que le premier objectif des établissements devienne aussi la scolarisation optimale, ce qui pourrait provoquer un retour au modèle « réforme » et la consolidation de ce modèle. Dans les pages qui suivent, ce danger est exploré à l'aide de données provenant de recherches récentes dans des établissements qui reçoivent des jeunes présentant des troubles de comportement.

\section{Les conflits d'objectif}

Les différents modèles de traitement décrits dans les pages précédentes sous-tendent des objectifs à poursuivre. Par exemple, dans une institution utilisant le modèle * carcéral », l'objectif sera de garder le client dans l'institution jusqu'à ce qu'il ait purgé sa peine, alors que dans une institution utilisant le modèle a thérapeutique » l'objectif sera d'aider les client à se comporter d'une 
façon plus « adéquate * en utilisant les divers moyens * thérapeutiques $\gg$.

L'observation d'institutions poursuivant plus d'un objectif a permis de constater que cette situation entraîne habituellement des conflits entre les groupes poursuivant des objectifs différents. Par exemple, dans les institutions pénitentiaires qui ont tenté de développer des programmes de resocialisation, on a observé des conflits importants entre le personnel responsable de la sécurité (objectif carcéral) et le personnel responsable de la resocialisation (objectif thérapeutique) (Maxim, 1976 ; Gendreau et Tremblay, 1977).

Une étude récente dans neuf Community Homes en Angleterre (Heal et Cawson, 1975) montre que les institutions du type " centre d'accueil » pour jeunes en difficulté d'adaptation sont loin d'être à l'épreuve de ces conflits d'objectifs entre différents groupes de membres du personnel.

Avant 1969, en Angleterre, les internats de rééducation pour adolescents délinquants étaient appelés Approved Schools. Ils étaient considérés comme des «écoles》 spéciales et le personnel était recruté surtout parmi les professionnels de l'enseignement. Le modèle d'intervention datait du dix-neuvième siècle et il peut être assimilé au modèle « réforme » décrit plus haut. En 1969 le gouvernement britannique passait une loi (H.M.S.O., 1969) visant à rendre ces institutions mieux intégrées dans la communauté, moins centrées sur la dimension scolaire et répondant plus adéquatement aux besoins d'aide des jeunes (Home Office, 1968). Ils nommèrent ces institutions Community Homes.

L'étude de Heal et Cawson (1975) visait à identifier les problèmes que ces changements d'orientation avaient provoqués dans les institutions. Ils en concluent que plusieurs difficultés sont dues au conflit entre deux modèles d'organisation : le modèle "scolaire » et le modèle « aide à l'enfant » (child care, p. 72).

Les données de cette étude (Heal et Cawson, 1975) montrent des différences importantes entre les objectifs des enseignants et des éducateurs. Par exemple, les enseignants perçoivent que la planification du traitement du jeune est le septième en importance dans leur rôle alors que les éducateurs perçoivent cet objectif comme étant le troisième en importance (p. 75). Les enseignants perçoivent l'éducation (enseignement) des jeunes com- 
me étant le premier en importance dans leur rôle alors que les éducateurs perçoivent cet objectif comme étant le septième en importance (p. 80).

Au niveau du soin apporté aux jeunes, les enseignants et les éducateurs sont plus près d'un concensus (deuxième en importance pour les enseignants et premier pour les éducateurs - p. 80) ; mais il semble que ce concensus concernant le rôle auprès du jeune soit la source d'un conflit important. Les enseignants expriment l'opinion qu'ils sont capables de satisfaire aux besoins des jeunes par les activités scolaires et ils perçoivent le travail en dehors des heures de classes comme étant de la surveillance (p. 93).

\section{Le climat social de linstitution}

Le climat social d'un milieu de traitement est généralement un bon indicateur de l'impact positif du milieu sur sa clientèle (Jesness, 1975 ; Moos, 1975 ; Tremblay, 1976). Le climat social du milieu est d'autre part (probablement en partie) une résultante de la qualité des relations entre les membres du personnel du milieu. Il apparaît raisonnable de croire que, là où les membres du personnel ne réussissent pas à s'entendre sur les objectifs à poursuivre et diminuent l'importance du rôle de l'autre, le climat social du milieu sera perçu comme tendu et l'impact sur les clients sera diminué.

Une étude du climat dans deux inst:tutions des environs de Londres devenues des Community Homes, après une longue tradition comme Approved School, permet d'illustrer ce probème. L'étude (Tremblay, 1976) visait à comparer l'évolution des adolescents délinquants en traitement dans les institutions québécoises et anglaises. Pour ce faire, le climat social de chacune des institutions a été évalué à l'aide du Correctional Institutions Environment Scale, de Moos (1973).

Dans une des institutions anglaises étudiées, l'organisation était demeurée sensiblement la même qu'au temps des Approved Schools. C'est-à-dire qu'il y avait un groupe d'enseignants qui recevait les enfants pour une journée à peu près normale d'activités scolaires dans un édifice réservé à cette fin sur le terrain de l'institution, alors que le groupe d'éducateurs s'occupait des levers, des repas, des soirées, des couchers et des fins de se- 
maine ${ }^{1}$. Il était donc facile d'identifier les deux groupes et, au cours de l'étude, la perception du climat social du milieu par chacun de ces groupes fut comparée à la perception des garçons.

La figure I présente les résultats de cette étude. On peut y noter non seulement la différence importante entre la perception des éducateurs et celle des enseignants, mais la perception des garçons (des adolescents délinquants) qui se révèle plus positive que celle des enseignants. Un observateur connaissant bien le milieu fit la remarque, en étudiant cette figure, que les garçons semblaient être pris entre deux feux.

La figure II montre les résultats d'une étude du climat social d'une institution qui tentait de diminuer les différences de rôles entre éducateurs et enseignants. Il est à remarquer que les différences de perception entre les éducateurs et les enseignants sont minimes en comparaison des résultats de la figure I. De plus, les enseignants ont, sur trois dimensions, une vision légèrement plus positive que celle des éducateurs, ce qui ne se produit pas dans l'institution présentée au tableau I (sauf sur la dimension \& contrôle du personnel > où les résultats sont presque toujours inversement proportionnels aux autres dimensions).

\section{Conclusion}

La mission MEQ - MAS (1974) vise la scolarisation optimale des jeunes dans les établissements du MAS. Cet objectif apparaît en soi louable mais chacun doit être conscient des dangers qu'il implique.

Premièrement le danger d'un retour à un modèle de traitement pour enfants en difficulté d'adaptation datant du dix-neuvième siècle. Il est particulièrement intéressant de constater qu'en même temps que le mouvement de scolarisation optimale se dessine au Québec, un pays reconnu comme à l'avant-garde dans le domaine des mesures sociales (l'Angleterre) tente de dé-scolariser les institutions pour jeunes en difficulté d'adaptation.

Deuxièmement, le danger de créer une situation de conflits d'objectif entre des groupes travaillant dans une même institution. Il est difficile d'éviter que des factions existent à l'intérieur

1. Les Community Homes crés depuis la loi de 1969 tendent à ne pas différencier enseignants et éducateurs au niveau de la tâche. Le personnel est réparti en petites équipes de traitement ou chacun est utilisé au niveau de ses compétences (cf. Tremblay, 1976, p. 139). 
FIGURE I

Scores bruts des éducateurs, enseignants et garçons du Community Home $I$ ait Correctional Institutions Environment Scale
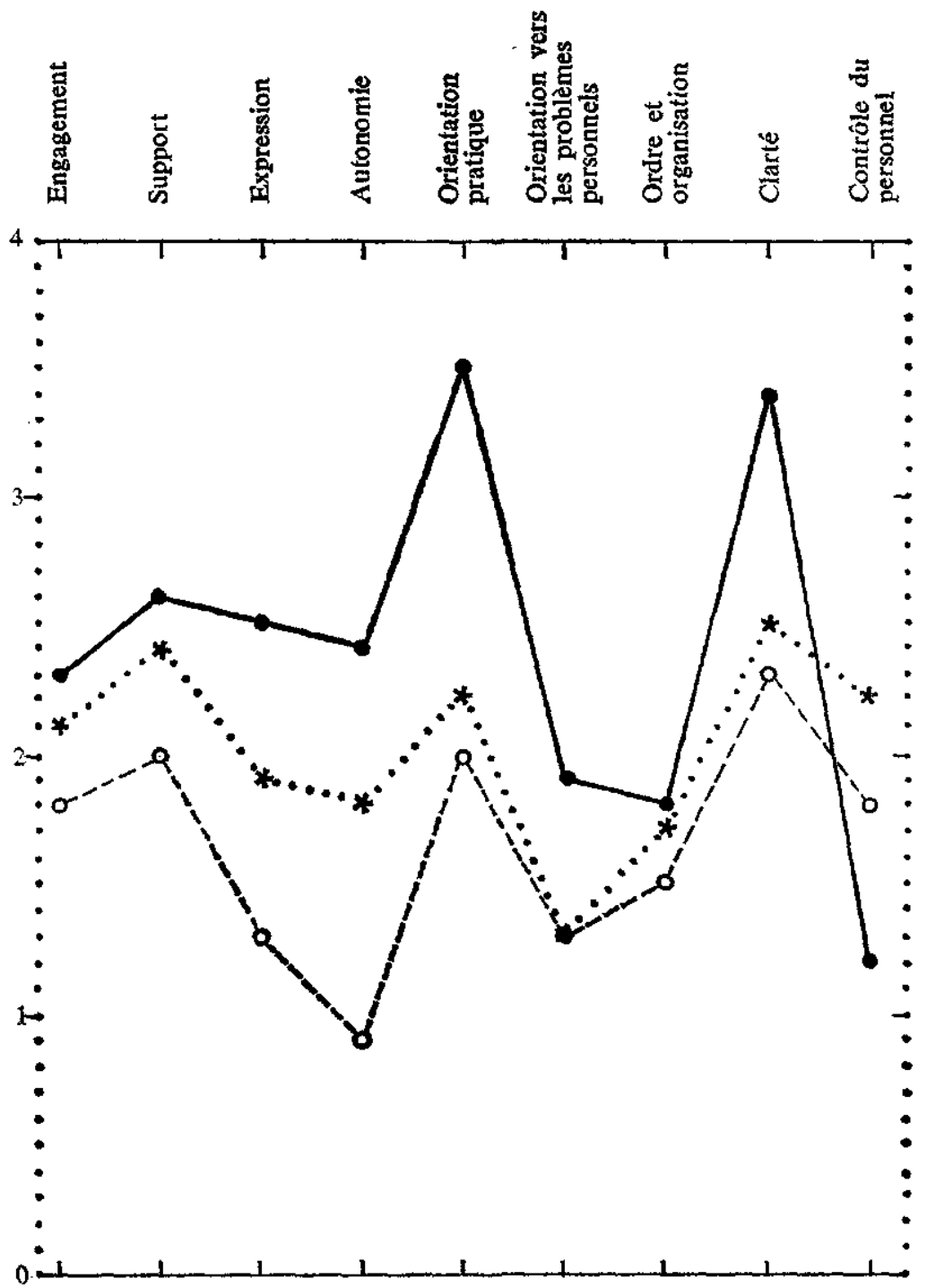

Educateurs $(\mathrm{N}=12)$

Enseignants $(\mathrm{N}=8)$

Garçons $(\mathbf{N}=64)$

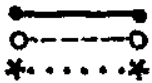




\section{FIGURE II}

Scores bruts des édicateurs, enseignants et garçons du Community Home 11 au Correctional Institutions Environment Scale
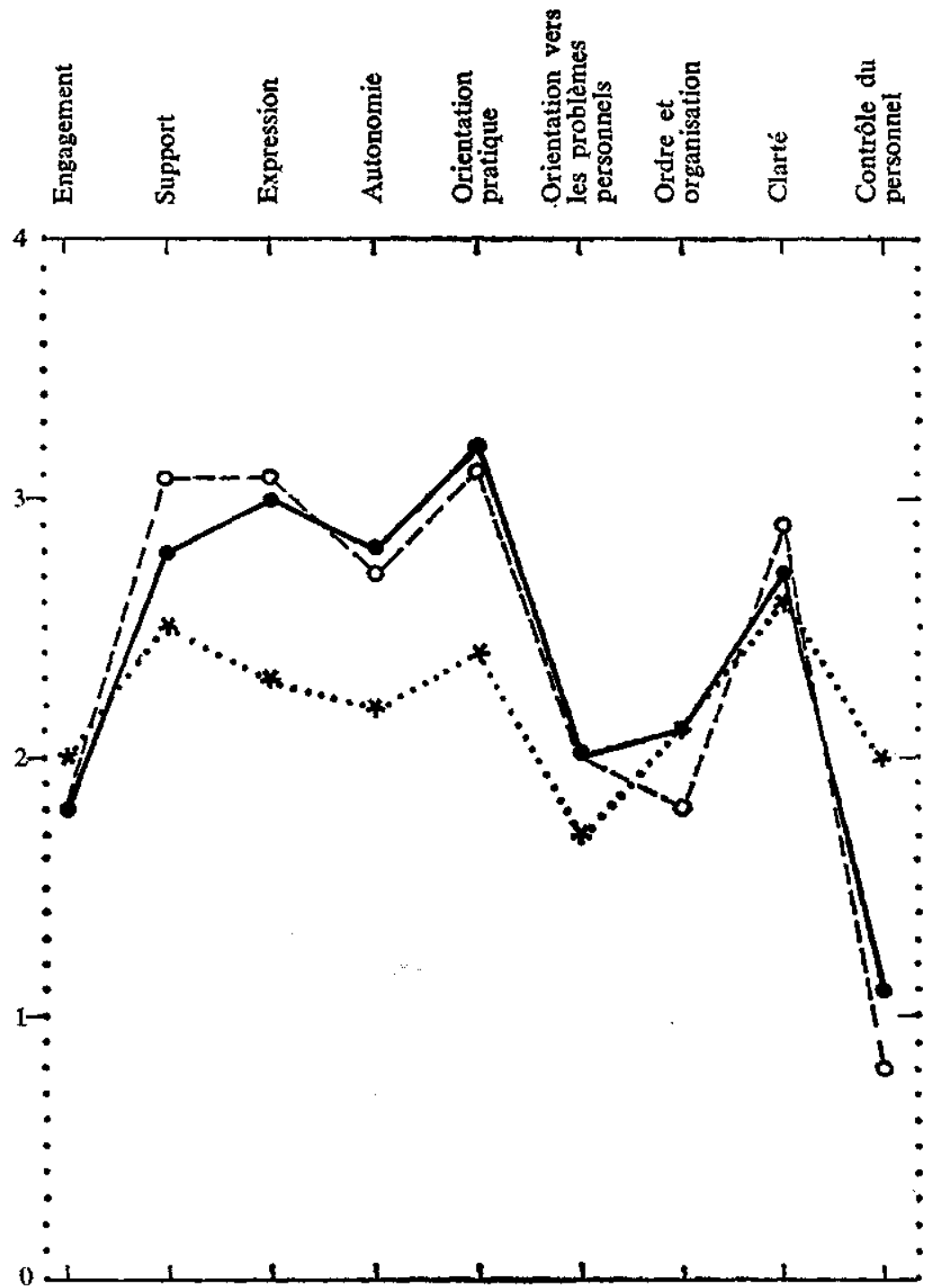

Educateurs $(\mathrm{N}=23)$

Enseignants $(N=9)$

Garçons $(N=42)$

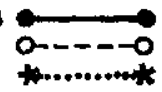


d'institutions, mais il serait peu raisonnable de créer des situations qui augmenteraient les chances de voir ces conflits se développer. Le "Guide \& de la mission MEQ - MAS propose que le personnel et les programmes (enseignement - réadaptation) relèvent de deux instances différentes (commission scolaire - établissement) et qu'un comité permanent agisse comme coordonnateur entre les deux instances (MEQ - MAS, 1974, p. 17-18 et 24). Cette organisation paraît créer la situation idéale pour que deux groupes bien identifiés se livrent une guerre d'objectifs et de moyens sur le dos des clients, à moins que les comités permanents soient efficaces au point de réussir ce qui n'a pas été obtenu ailleurs dans des situations moins difficiles.

Troisièmement, le danger de compromettre le climat social des institutions par la création d'une situation où enseignants et éducateurs se percevraient comme deux entités rivales.

Il est difficile de ne pas souhaiter la scolarisation optimale des jeunes dans les établissements du MAS, mais il est encore plus difficile d'accepter que cet objectif soit poursuivi aux dépens de l'aide dont ces enfants ont besoin. Les éducateurs et les enseignants réussiront à unir leurs efforts s'ils se perçoivent comme des confrères, des coéquipiers. La formation de petites équipes d'enseignants-éducateurs, chacune responsable d'un petit groupe d'enfants, aurait peut-être plus de chance d'assurer une meilleure coordination des objectifs de l'enseignement et de la réadaptation en fonction des besoins de chacun des jeunes, qu'un comité de coordination aux paliers supérieurs.

\section{BIBLIOGRAPHIE}

BATSHAW, M.G. et al. (1975), Rapport du comité d'étude sur la réadaptation des enfants et adolescents placés en centre d'accueil, Québec, ministère des Affaires sociales.

DUNLOP, A.B. (1974), The Approved School Experience, London, H.M.S.O.

GENDREAU, G., TREMBLAY, R. (1977), Rapport d'expertise: Institution de Cowansville, Ottawa, Service canadien des pénitenciers.

HEAL, K., CAWSON, P. (1975), \& Organization and Change in children's Institutions \$, in Tizard, J. et al. (Eds.) (1975), Varieties of residential experience, London, Routledge et Kegan Paul.

H.M.S.O. (1969), Children and Young persons act 1969, London, H.M.S.O. HOME OFFICE (1968), Children in trouble, London, H.M.S.O. 
HOOD, R.G., SPARKS, R. (1970), Key issues in criminology, London, Weidenfeld and Nicolson.

JESNESS, C.F. (1975), Comparative effectiveness of behavior modification and transactional analysis programs for delinquents 3, Journal of Consulting and Clinical Psychology, 43, 6, p. 758-779.

LEHMAN, P.E. (1972), The medical model of treatment : historical development of an archaic standard s, Crime and Delinquency, 18, 2, 204-212.

MAXIM, P. (1976), * Treatment-custody staff conflicts in correctional institutions : a re-analysis s, Revue canadienne de criminologie, 18, 4, p. 379-386.

MEQ-MAS (1974), Guide élaboré conjointement par le ministère des Affaires sociales et le ministère de l'Education pour assurer la scolarisation optimale des enfants des établissements du ministère des Affaires sociales, Québec, ministère des Affaires sociales, ministère de l'Education.

MILLHAM, S., BULLOCK, R., CHERRET, P. (1973), « Can we legislate for care? ?, Special Education, 62, 4, p. 10-13.

MOOS, R.H. (1973), Correctional Institutions Environment Scale Manual, Palo Alto, California, Social Ecology Laboratory, Department of Psychiatry, Stanford University.

MOOS, R.H. (1975), Evaluating Correctional and Community Settings, London, John Wiley and Sons.

SANDERS, W.B. (1970), Juvenile offenders for a thousand years, Chapel Hill, University of North Carolina Press.

STREET, D., VINTER, R.D., PERROW, G. (1966), Organization for treatment : A comparative study of institutions for delinquents, New York, Free Press.

TREMBLAY, R.E. (1976), A psycho-educational study of juvenile delinquents during residential treatment, Thèse de doctorat inedite, Institut d'éducation, Université de Londres.

WOLFENSBERGER, W. (1972), The principle of Normalization in human services, Toronto, National Institute on Mental Retardation. 